\title{
THE FINITENESS OF CO-ASSOCIATED PRIMES OF LOCAL HOMOLOGY MODULES
}

\author{
Tran TuAn NAM
}

\begin{abstract}
Let $M$ be a semi-discrete linearly compact module over a commutative noetherian ring $R$ and $i$ a non-negative integer. We show that the set of co-associated primes of the local homology $R$-module $H_{i}^{I}(M)$ is finite in either of the following cases: (i) The $R$-modules $H_{j}^{I}(M)$ are finite for all $j<i$; (ii) $I \subseteq \operatorname{Rad}\left(\operatorname{Ann}_{R}\left(H_{j}^{I}(M)\right)\right)$ for all $j<i$. By Matlis duality we extend some results for the finiteness of associated primes of local cohomology modules $H_{I}^{i}(M)$.
\end{abstract}

\section{Introduction}

In [10] Huneke asked the question: If $M$ is finite, is the number of associated primes of local cohomology modules $H_{I}^{i}(M)$ always finite? there is a similar question in local homology theory: When is the set of co-associated primes of local homology modules finite? In $[4,3.1] \mathrm{N}$. T. Cuong and the author defined the local homology modules $H_{i}^{I}(M)$ of $M$ with respect to $I$ by

$$
H_{i}^{I}(M)=\underset{t}{\lim } \operatorname{Tor}_{i}^{R}\left(R / I^{t} ; M\right) \text {. }
$$

This definition is in some sense dual to A. Grothendieck's definition of local cohomology modules. Next, in [6] the authors studied local homology modules for the category of linearly compact $R$-modules, which contains strictly all Artinian modules and also finite $R$-modules when $R$ is a complete local ring. It should be noted that in the case of linearly compact modules, this definition of local cohomology modules is coincident with the definition of J. P. C. Greenlees and J. P. May $[9,2.4]$. We showed in $[5,4.5]$ that if $M$ is a semi-discrete linearly compact $R$-module and the local homology $R$-modules $H_{0}^{I}(M), H_{1}^{I}(M), \ldots$,

2000 Mathematics subject classification. Local cohomology 13D45, Homological functors on modules 16E30, Topological rings and modules 13J99.

Key words. linearly compact module, local homology, local cohomology, co-associated prime, associated prime.

The Abdus Salam International Centre for Theoretical Physics Strada Costiera 11, 34014 Trieste, Italy.

Received November 14, 2005; revised April 13, 2006. 
$H_{i-1}^{I}(M)$ are artinian, then the set $\operatorname{Coass}_{R}\left(H_{i}^{I}(M)\right)$ is finite. In Theorem 3.1 we show that the set of co-associated primes of the local homology module $H_{i}^{I}(M)$ is finite in either of the following cases:

(i) The $R$-modules $H_{j}^{I}(M)$ are finite for all $j<i$;

(ii) $I \subseteq \operatorname{Rad}\left(\operatorname{Ann}_{R}\left(H_{j}^{I}(M)\right)\right)$ for all $j<i$.

Using Matlis duality, we get the finiteness for associated primes of local cohomology modules (Corollary 3.6) which is also an extension of [2, 2.2], [12, Th. B] and $[15,5.5]$. It should be noted that the finiteness of the $\operatorname{sets}^{\operatorname{Coass}}{ }_{R}\left(H_{i}^{I}(M)\right)$ and $\operatorname{Ass}_{R}\left(H_{I}^{i}(M)\right)$ is closely related to the local-global-principle for finiteness dimensions of $\mathrm{G}$. Faltings [8]. Throughout this paper, $R$ is a commutative noetherian ring with non-zero identity and has a topological structure.

\section{Premilinaries}

We begin by recalling briefly definitions and basic properties of linearly compact modules and local homology modules that we shall use.

Let $M$ be a topological $R$-module. $\quad M$ is said to be linearly topologized if $M$ has a base of neighborhoods of the zero element $\mathscr{M}$ consisting of submodules. $M$ is called Hausdorff if the intersection of all the neighborhoods of the zero element is 0 . A Hausdorff linearly topologized $R$-module $M$ is said to be linearly compact if $\mathscr{F}$ is a family of closed cosets (i.e., cosets of closed submodules) in $M$ which has the finite intersection property, then the cosets in $\mathscr{F}$ have a non-empty intersection (see [13]).

It is clear that artinian $R$-modules are linearly compact and discrete. If $(R, \mathrm{~m})$ is a complete ring, then finite $R$-modules are also linearly compact and discrete.

We know that the inverse limit $\lim _{t}$ is a left exact functor on inverse systems of modules. However, if $\left\{M_{t}\right\}$ is an inverse system of linearly compact modules with continuous homomorphisms, we have the following lemma.

Lemma 2.1 (see $[6,2.4])$. Let

$$
0 \rightarrow\left\{M_{t}\right\} \rightarrow\left\{N_{t}\right\} \rightarrow\left\{P_{t}\right\} \rightarrow 0
$$

be a short exact sequence of inverse systems of $R$-modules. If $\left\{M_{t}\right\}$ is an inverse system of linearly compact modules with continuous homomorphisms, then the sequence of inverse limits

$$
0 \rightarrow \underset{t}{\lim } M_{t} \rightarrow \underset{t}{\lim } N_{t} \rightarrow \underset{t}{\lim } P_{t} \rightarrow 0
$$

is exact.

Let $I$ be an ideal of $R$, the $i$-th local homology module $H_{i}^{I}(M)$ of an $R$ module $M$ with respect to $I$ is defined in [4] by

$$
H_{i}^{I}(M)=\lim _{t} \operatorname{Tor}_{i}^{R}\left(R / I^{t}, M\right)
$$


THE FINITENESS OF CO-ASSOCIATED PRIMES OF LOCAL HOMOLOGY MODULES 385

Denote by $\Lambda_{I}(M)=\lim _{t} M / I^{t} M$ the $I$-adic completion of $M$, then $H_{0}^{I}(M) \cong$ $\Lambda_{I}(M)$. In case $M \overleftarrow{\text { is }}$ a finite $R$-module, $H_{i}^{I}(M)=0$ for all $i>0$ (see [4, 3.2(ii)]). It should be noted that this definition of local homology modules is coincident with the definition of J. P. C. Greenlees and J. P. May [9] in the case of linearly compact modules. The local homology modules $H_{i}^{I}(M)$ of a linearly compact $R$-module $M$ are also linearly compact $R$-modules. Moreover, every short exact sequence of linearly compact $R$-modules induces a long exact sequence of local homology modules (see $[6,4.6])$. We have some basic properties of local homology modules.

Lemma 2.2 (see [4, 3.3(i)]). Let $M$ be an R-module. Then for all $i \geq 0$, the local homology module $H_{i}^{I}(M)$ is I-separated, i.e.,

$$
\bigcap_{s>0} I^{s} H_{i}^{I}(M)=0
$$

Denote by $E(R / \mathfrak{m})$ the injective envelope of $R / \mathfrak{m}$, the Matlis dual of an $R$-module $M$ is the module $D(M)=\operatorname{Hom}_{R}(M, E(R / \mathrm{m}))$. Let $H_{I}^{i}(M)$ be the $i$-th local cohomology module of $M$ respect to $I$. We have the following dual formula.

Lemma 2.3 (see $[4,3.3(\mathrm{ii})]$ ). Let $(R, \mathrm{~m})$ be a local noetherian ring and $M$ an $R$-module. Then for all $i \geq 0$,

$$
H_{i}^{I}(D(M)) \cong D\left(H_{I}^{i}(M)\right) .
$$

In case $M$ is a linearly compact module, we have the following properties.

Lemma 2.4 (see $[6,4.8]$ ). Let $M$ be a linearly compact $R$-module. Then

$$
H_{i}^{I}\left(\bigcap_{t>0} I^{t} M\right) \cong \begin{cases}0, & i=0 \\ H_{i}^{I}(M), & i>0 .\end{cases}
$$

A Hausdorff linearly topologized $R$-module $M$ is called semi-discrete if every submodule of $M$ is closed. Thus a discrete $R$-module is semi-discrete. The class of semi-discrete linearly compact $R$-modules contains all artinian $R$-modules. Moreover, it also contains all finite modules in case $R$ is a complete local ring (see $[13,7.3])$.

Lemma 2.5 (see $[6,5.2])$. Let $M$ be a semi-discrete linearly compact $R$ module. Then $H_{0}^{I}(M)=0$ if and only if $x M=M$ for some $x \in I$.

\section{The finiteness of co-associated primes}

We first recall the concept of co-associated primes. A module is called cocyclic if it is a submodule of $E(R / \mathrm{m})$ for some maximal ideal $\mathrm{m}$ of $R$. A 
prime ideal $\mathfrak{p}$ is called co-associated to a non-zero $R$-module $M$ if there is a cocyclic homomorphic image $T$ of $M$ with $\mathfrak{p}=\operatorname{Ann}_{R} T$ [16]. Note that this concept of co-associated primes is equivalent with the concepts of $\mathrm{L}$. Chambless [3] and H. Zöschinger [19]. The set of co-associated primes of $M$ is denoted by $\operatorname{Coass}_{R}(M) . \quad M$ is called $\mathfrak{p}$-coprimary if $\operatorname{Coass}_{R}(M)=\{\mathfrak{p}\} . \quad$ If $M$ is a semidiscrete linearly compact $R$-module, then the set $\operatorname{Coass}_{R}(M)$ is finite (see $[6,3.4]$ ).

We proved in $[5,4.5]$ that if $M$ is a semi-discrete linearly compact $R$ module and the $R$-modules $H_{0}^{I}(M), H_{1}^{I}(M), \ldots, H_{i-1}^{I}(M)$ are artinian, then the set $\operatorname{Coass}_{R}\left(H_{i}^{I}(M)\right)$ is finite. We show an other finiteness result for the set $\operatorname{Coass}_{R}\left(H_{i}^{I}(M)\right)$. Note that in Theorem 3.1 the $\operatorname{ring} R$ is not necessary local.

THEOREM 3.1. Let $M$ be a semi-discrete linearly compact $R$-module and $i$ a non-negative integer. The set of co-associated primes of the local homology $R$-module $H_{i}^{I}(M)$ is finite in either of the following cases:

(i) The $R$-modules $H_{j}^{I}(M)$ are finite for all $j<i$;

(ii) $I \subseteq \operatorname{Rad}\left(\operatorname{Ann}_{R}\left(H_{j}^{I}(M)\right)\right)$ for all $j<i$.

To prove Theorem 3.1, the following lemmas are necessary.

If $0 \rightarrow N \rightarrow M \rightarrow K \rightarrow 0$ is an exact sequence of $R$-modules, then Coass ${ }_{R}(K)$ $\subseteq \operatorname{Coass}_{R}(M) \subseteq \operatorname{Coass}_{R}(N) \cup \operatorname{Coass}_{R}(K)$ (see [16, 1.10]). In general we do not have the equality $\operatorname{Coass}_{R}(M)=\operatorname{Coass}_{R}(N) \cup \operatorname{Coass}_{R}(K)$. However, in the following lemma we show that the equality is true in case $K$ is a finite module.

Lemma 3.2. Let $0 \rightarrow N \rightarrow M \rightarrow K \rightarrow 0$ be an exact sequence of R-modules. If $K$ is a finite $R$-module, then

$$
\operatorname{Coass}_{R}(M)=\operatorname{Coass}_{R}(N) \cup \operatorname{Coass}_{R}(K) .
$$

Proof. Without loss of generality we may assume that $N$ is a submodule of $M$, then $K \cong M / N$ and $\operatorname{Coass}_{R}(K)=\operatorname{Coass}_{R}(M / N)$. From the short exact sequence

$$
0 \rightarrow N \rightarrow M \rightarrow M / N \rightarrow 0
$$

we have

$$
\operatorname{Coass}_{R}(M) \subseteq \operatorname{Coass}_{R}(N) \cup \operatorname{Coass}_{R}(M / N)
$$

by $[16,1.10]$. Now let $\mathfrak{p} \in \operatorname{Coass}_{R}(N), \mathfrak{p} \notin \operatorname{Coass}_{R}(M / N)$. We have to prove that $\mathfrak{p} \in \operatorname{Coass}_{R}(M)$.

If $\mathfrak{p}$ is a maximal ideal, then there is an artinian homomorphic image $N / B$ of $N$ such that $\mathfrak{p}=\operatorname{Ann}(N / B)$ by $[16,1.6]$. Thus $N / B$ has finite length by $[17$, 7.30]. From the following short exact sequence

$$
0 \rightarrow N / B \rightarrow M / B \rightarrow M / N \rightarrow 0,
$$

we have the fact that $M / B$ is finite. Combining [16, 2.6], [16, 2.7] and [16, 2.10] yields $\operatorname{Coass}_{R}(M / B)=\operatorname{Coass}_{R}(N / B) \cup \operatorname{Coass}_{R}(M / N)$. Therefore $\mathfrak{p} \in$ $\operatorname{Coass}_{R}(M / B)$ and then $\mathfrak{p} \in \operatorname{Coass}_{R}(M)$. 
In case $p$ is not a maximal ideal, by $[16,1.16]$, there is a sumirreducible homomorphic image $N / C$ of $N$ with $\mathfrak{p}=\{x \in R \mid x(N / C) \neq N / C\}$ and $\operatorname{Coass}_{R}(N / C)=\{\mathfrak{p}\}$. Thus we have the following short exact sequence

$$
0 \rightarrow N / C \rightarrow M / C \rightarrow M / N \rightarrow 0
$$

and hence $\operatorname{Coass}_{R}(M / C) \subseteq \operatorname{Coass}_{R}(N / C) \cup \operatorname{Coass}_{R}(M / N)=\{\mathfrak{p}\} \cup \operatorname{Coass}_{R}(M / N)$. Since $p$ is not a maximal ideal, $N / C$ is not a finite $R$-module, so $M / C$ is also not a finite $R$-module. It should be noted that the co-associated primes of a finite module are only maximal ideals. Therefore $\mathfrak{p} \in \operatorname{Coass}_{R}(M / C) \subseteq \operatorname{Coass}_{R}(M)$ and the proof is complete.

Corollary 3.3. Let $0 \rightarrow N \rightarrow M \rightarrow K \rightarrow 0$ be an exact sequence of $R$ modules. If $K$ is a finite $R$-module and $\operatorname{Coass}_{R}(M)$ is finite, then $\operatorname{Coass}_{R}(N)$ is also finite.

Proof. By 3.2, we have $\operatorname{Coass}_{R}(M)=\operatorname{Coass}_{R}(N) \cup \operatorname{Coass}_{R}(K)$. Since $\operatorname{Coass}_{R}(M)$ is finite, $\operatorname{Coass}_{R}(N)$ is also finite.

Lemma 3.4. Let $M$ be a I-separated linearly compact R-module. If there is an element $x \in I$ such that $\operatorname{Coass}_{R}(M / x M)$ is finite, then $\operatorname{Coass}_{R}(M)$ is finite.

Proof. Let $\mathfrak{p} \in \operatorname{Coass}_{R}(M)$, then there is an artinian quotient $R$-module $M / N$ of $M$ with $\mathfrak{p}=\operatorname{Ann}(M / N)$. Hence there is a positive integer $n$ such that $x^{n}(M / N)=x^{t}(M / N)$ for all $t \geq n$. Since $\bigcap_{t>0} x^{t} M=0$, we have $x^{n}(M / N)=\bigcap_{t>0} x^{t}(M / N)=0$. Thus $x^{n} M \subseteq N$, so $M / N$ may be considered as an artinian quotient $R$-module of $M / x^{n} M$. Therefore $\mathfrak{p} \in \operatorname{Coass}_{R}\left(M / x^{n} M\right)$, that means $\operatorname{Coass}_{R}(M) \subseteq \operatorname{Coass}_{R}\left(M / x^{n} M\right)$. Thus we need only to show that $\operatorname{Coass}_{R}\left(M / x^{n} M\right)$ is finite. Indeed, from the epi-morphim $M / x M \stackrel{\cdot x}{\rightarrow} x M / x^{2} M$ we have $\operatorname{Coass}_{R}\left(x M / x^{2} M\right) \subseteq \operatorname{Coass}_{R}(M / x M)$ is finite. Now, the short exact sequence

$$
0 \rightarrow x M / x^{2} M \rightarrow M / x^{2} M \rightarrow M / x M \rightarrow 0
$$

gives $\quad \operatorname{Coass}_{R}\left(M / x^{2} M\right) \subseteq \operatorname{Coass}_{R}\left(x M / x^{2} M\right) \cup \operatorname{Coass}_{R}(M / x M)$. Therefore $\operatorname{Coass}_{R}\left(M / x^{2} M\right)$ is finite. By induction we get $\operatorname{Coass}_{R}\left(M / x^{n} M\right)$ is finite. This finishes the proof.

Now we can prove Theorem 3.1.

Proof of Theorem 3.1. (i) We prove by induction on $i$. When $i=0$, for all positive integers $t$ the canonical epi-morphisms $M \rightarrow M / I^{t} M$ induces an epimorphism $M \rightarrow \Lambda_{I}(M)$ by 2.1. Then we have $\operatorname{Coass}_{R}\left(\Lambda_{I}(M)\right) \subseteq \operatorname{Coass}_{R}(M)$ which is finite by $[6,3.4]$, because $M$ is a semi-discrete linearly compact $R$-module. 
Let $i>0$. Combining 2.4 with 2.5, we may assume that there is an element $x \in I$ such that $x M=M$. Then the short exact sequence of linearly compact modules

$$
0 \rightarrow 0: M x \stackrel{x}{\rightarrow} M \rightarrow 0
$$

induces an exact sequence of local homology modules

$$
\cdots H_{i}^{I}(M) \stackrel{x}{\rightarrow} H_{i}^{I}(M) \stackrel{\delta}{\rightarrow} H_{i-1}^{I}\left(0:_{M} x\right) \stackrel{\alpha}{\rightarrow} H_{i-1}^{I}(M) .
$$

If $0::_{M} x=0$, then $H_{i}^{I}(M)=x H_{i}^{I}(M)=\bigcap_{t>0} x^{t} H_{i}^{I}(M)=0$ for all $i \geq 0$ by 2.2 . We now assume that $0::_{M} x \neq 0$. Since the $R$-modules $H_{j}^{I}(M)$ are finite for all $j<i$, the $R$-modules $H_{j}^{I}\left(0:_{M} x\right)$ are also finite for all $j<i-1$. Then $\operatorname{Coass}_{R}\left(H_{i-1}^{I}\left(0:_{M} x\right)\right)$ is finite. Now we have $H_{i-1}^{I}\left(0:_{M} x\right) / \operatorname{Im} \delta=H_{i-1}^{I}\left(0:_{M} x\right) /$ $\operatorname{ker} \alpha \cong \operatorname{Im} \alpha \subseteq H_{i-1}^{I}(M)$. Because $H_{i-1}^{I}(M)$ is finite, $H_{i-1}^{I}\left(0:_{M} x\right) / \operatorname{Im} \delta$ is also finite. Thus $\operatorname{Coass}_{R} \operatorname{Im} \delta$ is finite by 3.3. That means $\operatorname{Coass}_{R}\left(H_{i}^{I}(M) / x H_{i}^{I}(M)\right)$ is finite. Therefore $\operatorname{Coass}_{R}\left(H_{i}^{I}(M)\right)$ is finite by 3.4.

(ii) The case $i=0$ was proved in (i).

Let $i>0$. Denote by $L(M)$ the sum of all artinian submodules of $M$, then $L(M)$ is artinian by $[19,1(\mathrm{~L} 5)]$. From [6, 5.4] we have the isomorphism $H_{j}^{I}(M) \cong H_{j}^{I}(L(M))$ for all $j>0$ and the short exact sequence

$$
0 \rightarrow H_{0}^{I}(L(M)) \rightarrow H_{0}^{I}(M) \rightarrow H_{0}^{I}(M / L(M)) \rightarrow 0 .
$$

Then for all $j<i$ we have $I \subseteq \operatorname{Rad}\left(\operatorname{Ann}_{R}\left(H_{j}^{I}(L(M))\right)\right)$, hence $H_{j}^{I}(L(M))$ are artinian by $[4,4.7]$. Therefore $\operatorname{Coass}_{R}\left(H_{i}^{I}(M)\right)=\operatorname{Coass}_{R}\left(H_{i}^{I}(L(M))\right)$ is finite by $[5,4.5]$. The proof is complete.

Remark 3.5. In case $M$ is semi-discrete linearly compact, the condition $I \subseteq \operatorname{Rad}\left(\operatorname{Ann}_{R}\left(H_{j}^{I}(M)\right)\right)$ for all $j<i$ may not follow $H_{j}^{I}(M)$ are artinian for all $j<i$. For example, let $(R, \mathfrak{m})$ be a complete local noetherian ring and $N$ a finite $R$-module such that the module $M=N / I N$ is not artinian. Then $H_{0}^{I}(M) \cong \Lambda_{I}(M) \cong M$. Thus $I \subseteq \operatorname{Rad}\left(\operatorname{Ann}_{R}\left(H_{0}^{I}(M)\right)\right)$, but $H_{0}^{I}(M)$ is not artinian. Therefore (ii) is an extension of $[5,4.5]$.

We now use Matlis duality to get a finiteness result for associated primes of local cohomology module. In Corollary $3.6(R, \mathrm{~m})$ is a local ring and the topology of $R$ is the m-adic topology.

Corollary 3.6. Let $(R, \mathrm{~m})$ be a complete local ring with the $\mathrm{m}$-adic topology and $M$ a semi-discrete linearly compact $R$-module. Let $i$ be a non-negative integer. The set of associated primes of the local cohomology module $H_{I}^{i}(M)$ is finite in either of the following cases:

(i) The modules $H_{I}^{j}(M)$ are artinian for all $j<i$;

(ii) $I \subseteq \operatorname{Rad}\left(\operatorname{Ann}_{R}\left(H_{I}^{j}(M)\right)\right)$ for all $j<i$. 
Proof. We have the following isomorphism by 2.3

$$
D\left(H_{I}^{j}(M)\right) \cong H_{j}^{I}(D(M)) \text {. }
$$

(i) It follows from $[18,3.4 .12]$ that $R$-modules $H_{j}^{I}(D(M))$ are finite for all $j<i$. Moreover, $D(M)$ is also a semi-discrete linearly compact $R$-module by $[13,5.8,9.13]$. Then the set $\operatorname{Coass}_{R}\left(H_{i}^{I}(D(M))\right)$ is finite by 3.1 , so is Coass $_{R} D\left(H_{I}^{i}(M)\right)$. On the other hand, in virtue of $[16,1.18]$ we have $\operatorname{Ass}_{R}\left(H_{I}^{i}(M)\right) \subseteq \operatorname{Coass}_{R}\left(D\left(H_{I}^{i}(M)\right)\right)$. Therefore the set $\operatorname{Ass}_{R}\left(H_{I}^{i}(M)\right)$ is finite.

(ii) We have $\operatorname{Ann}_{R}\left(H_{j}^{I}(D(M))\right)=\operatorname{Ann}_{R}\left(D\left(H_{I}^{j}(M)\right)\right)=\operatorname{Ann}_{R}\left(H_{I}^{j}(M)\right)$ by [18, 3.4.2]. Then $I \subseteq \operatorname{Rad}\left(\operatorname{Ann}_{R}\left(H_{j}^{I}(D(M))\right)\right.$ for all $j<i$ and $D(M)$ is a semidiscrete linearly compact $R$-module. It follows $\operatorname{Coass}_{R}\left(H_{i}^{I}(D(M))\right)$ is finite, so is $\operatorname{Coass}_{R}\left(D\left(H_{I}^{i}(M)\right)\right)$. Hence $\operatorname{Ass}_{R}\left(H_{I}^{i}(M)\right) \subseteq \operatorname{Coass}_{R}\left(D\left(H_{I}^{i}(M)\right)\right)$ is finite. $\mathrm{B}(\beta)]$.

The following corollary is a result of $[2,2.2],[15,5.5]$ and $[12$, Theorem

Corollary 3.7 (see $[2,2.2]$, [12, Theorem $\mathrm{B}(\beta)]$ and $[15,5.5]$ ). Let $M$ be a finite $R$-module over a local ring $(R, \mathrm{~m})$ and $i$ a non-negative integer. The set of associated primes of the local cohomology module $H_{I}^{i}(M)$ is finite in either of the following cases:

(i) The modules $H_{I_{j}}^{j}(M)$ are artinian for all $j<i$;

(ii) The modules $H_{I}^{j}(M)$ are finite for all $j<i$.

Proof. From [1, 4.3.2] we have an isomorphism of $\hat{R}$-modules

$$
H_{I}^{i}(M) \otimes_{R} \hat{R} \cong H_{I \hat{R}}^{i}\left(M \otimes_{R} \hat{R}\right),
$$

in which $M \otimes_{R} \hat{R}$ is a finite $\hat{R}$-module. On the other hand the natural homomorphism of rings $f: R \rightarrow \hat{R}$ induces a map $f^{*}: \operatorname{Spec}(\hat{R}) \rightarrow \operatorname{Spec}(R)$. Moreover, it follows from $[14,9 . \mathrm{B}]$ that $\operatorname{Ass}_{R}\left(H_{I}^{i}(M)\right)=f^{*}\left(\operatorname{Ass}_{\hat{R}}\left(H_{I \hat{R}}^{i}\left(M \otimes_{R} \hat{R}\right)\right)\right.$. Thus, replacing $M$ by $M \otimes_{R} \hat{R}$, we may assume without any loss of generality that $(R, \mathfrak{m})$ is a complete local ring. Then $M$ is semi-discrete linearly compact by $[13,7.3]$. On the other hand the hypothesis (ii) follows that $I \subseteq$ $\operatorname{Rad}\left(\operatorname{Ann}_{R}\left(H_{I}^{j}(M)\right)\right)$ for all $j<i$ by [7, Theorem]. Therefore the result follows from 3.6.

Remark 3.8. When $M$ is semi-discrete linearly compact, the condition $I \subseteq \operatorname{Rad}\left(\operatorname{Ann}_{R}\left(H_{I}^{j}(M)\right)\right)$ for all $j<i$ may not follow $H_{I}^{j}(M)$ are finite for all $j<i$. For example, let $N$ be an artinian $R$-module such that the module $M=0:_{N} I$ is not finite. Then $H_{I}^{0}(M) \cong M$. Thus $I \subseteq \operatorname{Rad}\left(\operatorname{Ann}_{R}\left(H_{I}^{0}(M)\right)\right)$, but $H_{I}^{0}(M)$ is not finite.

Acknowledgments. The author would like to express his gratitude to prof. Nguyen Tu Cuong for his support and advice. We also acknowledge support by the Abdus Salam International Centre for Theoretical Physics, Trieste, Italy under the Post-doctoral fellowship. 


\section{REFERENCES}

[1] M. P. Brodmann AND R. Y. Sharp, Local cohomology: an algebraic introduction with geometric applications, Cambridge University Press, United Kingdom, 1998.

[2] M. P. Brodmann and A. L. Faghani, A finiteness result for associated primes of local cohomology modules, Proc. AMS 128 (2000), 2851-2853.

[ 3 ] L. Chambless, Coprimary decomposition, $N$-dimension and divisibility: application to Artinian modules, Comm. Algebra 9 (1981), 1131-1146.

[4] N. T. CuOng And T. T. Nam, The $I$-adic completion and local homology for Artinian modules, Math. Proc. Camb. Phil. Soc. 131 (2001).

[5] N. T. CuONG AND T. T. NAM, On the co-localization, co-support and co-associated primes of local homology modules, Vietnam J. Math. 29 (2001), 359-368.

[6] N. T. CuONG AND T. T. NAM, A local homology theory for linearly compact modules, ICTP preprint 120 (2004).

[7] G. Faltings, Über die Annulatoren localer Kohomologiegruppen, Arch. Math. 30 (1978), 473-476.

[8] G. Faltings, Der Endlichkeitssatz in der lokalen Kohomologie, Math. Ann. 255 (1981), 45-56.

[9] J. P. C. Greenless AND J. P. MaY, Derived functors of $I$-adic completion and local homology, J. Algebra 149 (1992), 438-453.

[10] C. Huneke, Problems on local cohomology, Free resolutions in commutative algebra and algebraic geometry, Sundance, Utah, 1990, Research notes in mathematics 2, Jones and Bartlett, Boston, MA, 1992, 93-108.

[11] C. U. Jensen, Les Foncteurs Dérivés de lim et leurs applications en théorie des modules, Springer-Verlag, Berlin-Heidelberg-New York, 1972.

[12] K. Khashyarmanesh and Sh. Salarian, On the associated primes of local cohomology modules, Comm. Algebra 27 (1999), 6191-6198.

[13] I. G. Macdonald, Duality over complete local rings, Topology 1 (1962), 213-235.

[14] H. Matsumura, Commutative algebra, Mathematics lecture note series 56, 2nd ed., Benjamin, 1980.

[15] L. T. Nhan, On geralized regular sequence and the finiteness for associated primes of local cohomology modules, Comm. Algebra 23 (2004), 1-15.

[16] S. Yassemi, Coassociated primes, Comm. Algebra 23 (1995), 1473-1498.

[17] R. Y. SHARP, Steps in commutative algebra, London math. soc. student texts 19, Cambridge University Press, 1990.

[18] J. Strooker, Homological questions in local algebra, London math. soc. lecture note ser. 145, Cambridge University Press, 1990.

[19] H. Zöschinger, Linear-Kompakte Moduln über noetherschen Ringen, Arch. Math. 41 (1983), 121-130.

Tran Tuan Nam

Department of Mathematics-Informatics

Ho Chi Minh University of Pedagogy

280 An Duong Vuong, District 5

Ho CHI Minh City

VIETNAM

E-mail: trtuannam@yahoo.com.vn,namtrantuan@dng.vnn.vn 